\title{
Enhancing Contrast of Weak Phase Objects Using a Zernike-Type Phase Plate in Phase Contrast TEM
}

\author{
E. Majorovits*, K. Nagayama**, and R. R. Schröder* \\ * Max-Planck-Institute for Medical Research, Jahnstr.29, 69120 Heidelberg, Germany \\ ** National Institute for Physiological Sciences, Myodaiji-cho, Okazaki 444-8585, Japan
}

One of the biggest challenges in modern electron microscopy is solving the problem of low image contrast of weak phase objects. In bright field imaging, contrast is enhanced by increased defocussing of the image, which leads to the problems of diminished contrast at high resolution and zero contrast for certain spatial frequencies. Maximal spatial frequency transfer bands are obtained at the Scherzer focus. However, the Scherzer focus is so close to the Gaussian focus that visibility of low spatial frequencies is greatly reduced (see figure 1, black curve, also figure 2a). This is particularly a problem for beam sensitive biological samples where the applicable electron dose is limited. Additionally the missing low spatial frequencies can lead to misinterpretations of images or artifacts in reconstructed 3D densities.

In light microscopy phase contrast imaging is a common method to visualize weak phase objects. This principle has recently be realized for TEM by the production of a Zernike-type phase plate [1]. The resulting phase contrast image shows a much higher contrast in the low spatial frequency range as compared to conventional bright field images (figure 1, grey curves). This results from the dominant cosine term in the corresponding Contrast Transfer Function CTF $\pi / 2(\mathrm{k})=$ $2[\cos (\mathrm{W}(\mathrm{k}))+\mathrm{A} \sin (\mathrm{W}(\mathrm{k}))]$ where $\mathrm{W}(\mathrm{k})$ denotes the wave aberration and A represents the contributing amplitude contrast.

Bright field and phase contrast images of filamentous actin embedded in vitrified ice were recorded using a LEO EFTEM $912 \Omega$. Phase contrast images were obtained with a Zernik-type phase plate positioned in the back focal plane of the objective lens of the microscope. This phase plate consisting of a thin layer of amorphous carbon film is described in [1]. Figure 1 shows a typical pair of bright field and phase contrast images demonstrating the effect of the phase plate on contrast transfer and object visibility. Both images were recorded close to focus. In the bright field image (fig. 2 a) no actin filaments are visible, there is no object contrast of the native protein (weak phase object). The white arrows indicate the position of the actin filaments, which become visible only in the phase contrast image (fig. 2 b).

The results presented show that phase contrast EM is a powerful means of visualizing weak phase objects, particularly unstained native biological objects, without the need of defocussing the objective lens and the resulting deteriorating effects. Artifacts due to missing low spatial frequencies are avoided. This new imaging technique should prove most powerful in combination with spherical aberration corrected TEM imaging. Combining phase plate phase contrast microscopy and spherical aberration corrected imaging results in an imaging mode facilitating a broad contrast transfer band without vanishing transfer at certain spatial frequencies. It will therefore be possible to recover the whole spatial frequency spectrum of any given object from only one image. This should greatly improve the SNR in images of beam senitive samples as all the cumulative electron dose can be 
applied to one image. In addition, image processing will be simplified as no contrast delocalization has to be corrected.

References

1. R. Danev, K. Nagayama, Ultramicroscopy, Vol. 88, No.4, pp. 243-252 (2001)

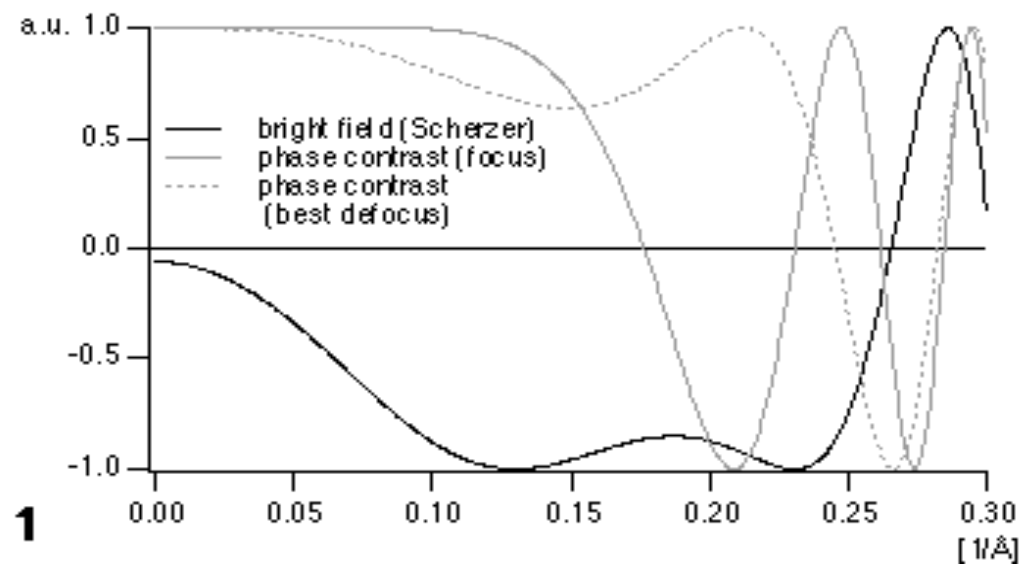

Figure 1 shows bright field and phase contrast contrast transfer functions (CTFs) at different defocus values (CTF parameters for a typical TEM such as LEO EFTEM $912 \Omega$ ). Bright field images recorded at the Scherzer focus yield a wide contrast transfer band but lack low spatial frequencies (black). Phase contrast images yield perfect contrast transfer at low spatial frequencies thus making objects visible. Recorded in focus, the CTF remains close to one for a wide frequency band (solid grey). Choosing the focus appropriately extends contrast transfer to higher frequencies.

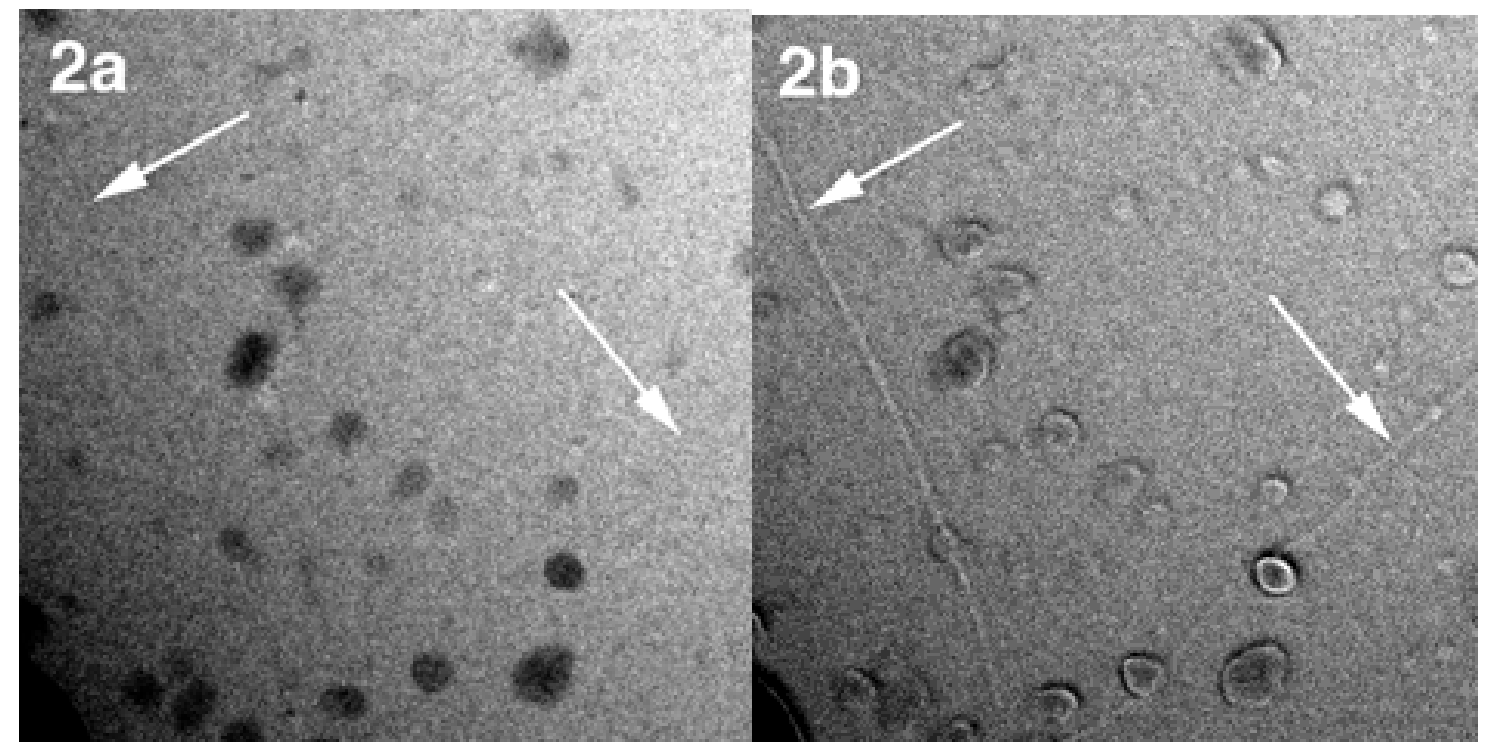

Figure 2 Bright field (a) and phase contrast (b) images of filamentous actin in vitrified ice recorded close to focus with a LEO EFTEM $912 \mathrm{~W}(120 \mathrm{kV}, \mathrm{Cs}=2.7 \mathrm{~mm})$. The phase contrast image was recorded with a Zernike-type phase plate inserted into the back focal plane of the objective lens. The actin filaments (white arrows) are only visible when recorded with the phase plate. 